\title{
The Optical Tamm States at the Interface between a Photonic Crystal and a Nanocomposite Containing Shelled Particles
}

\author{
S. Ya. Vetrov ${ }^{1,2}$, P. S. Pankin ${ }^{1}$ and I. V. Timofeev ${ }^{2,3}$ \\ ${ }^{1}$ Siberian Federal University, Institute of Engineering Physics and Radio Electronics, Krasnoyarsk, 660041 \\ Russia \\ ${ }^{2}$ Kirensky Institute of Physics, Russian Academy of Sciences, Siberian Branch, Krasnoyarsk, 660036 Russia \\ ${ }^{3}$ Siberian Federal University, Laboratory for Nonlinear Optics and Spectroscopy, Krasnoyarsk, 660041 Russia \\ e-mail:ppankin@sfu-kras.ru
}

\begin{abstract}
The optical Tamm states localized at the edges of a photonic crystal bounded by a nanocomposite on its one or both sides are investigated. The nanocomposite consists of spherical nanoparticles with a dielectric core and a metal shell, which are dispersed in a transparent matrix, and is characterized by the resonance permittivity. Spectra of transmission, reflection, and absorption of normally incident light by the investigated structures are calculated. The spectral manifestation of the Tamm states caused by negative values of the real part of the effective permittivity in the visible spectral range is studied. It is demonstrated that, along with the significantly extended photonic crystal band gap, the transmission spectrum contains an additional stopband caused by nanocomposite absorption near the resonance frequency. It is shown that the optical Tamm states can be implemented in two photonic crystal band gaps, each corresponding to a certain plasmon resonance frequency of the nanocomposite. It is established that the characteristic of the Tamm states localized at the edge of a photonic crystal significantly depend on the nanoparticle concentration in the nanocomposite film, on the thickness of the latter, and on the ratio between the particle core volume and the total particle volume. Waveguide modes formed by two bound Tamm plasmon polaritons at the interfaces between the photonic crystal and two nanocomposite layers are studied.
\end{abstract}

Keywords: photonic crystal, nanocomposite, optical Tamm state, shelled nanoparticles, effective permittivity.

PACS: $42.70 . \mathrm{Qs}$, 42.79.Gn, 78.66.Sq, 78.67.Bf

\section{Introduction}

In recent years, there has been an increased interest in a special type of the localized electromagnetic states excited at the normal incident of light onto a sample, which are called the optical Tamm states (OTSs) [1, 2]. Such states are analogous to the Tamm surface states in physics of condensed matter. They can be excited between two different photonic crystals (PCs) with overlapping band gaps [3] or between a PC and a medium 
with negative permittivity $\varepsilon[4,5]$. The surface electromagnetic wave at the interface between the photonic crystal and the medium with $\varepsilon<0$ is a single unit with the surface plasmon, i.e., oscillations of free electrons near the conductor surface. Such a bound mode of the radiation field and the surface plasmon excitation is called the surface plasmon polariton and is widely used in the visible and infrared spectral ranges for surface investigations. In the experiments, the OTS can be observed as a narrow peak in the transmission spectrum of a sample [6, 7].

Surface modes and OTSs can be used in sensors and optical switches [8], multichannel filters [9], Faraday- and Kerr-effect amplifiers [7, 10], organic solar cells [11], and absorbers [12].

Photonic crystals with nanostructured metal-dielectric inclusions offer new opportunities for manipulating light [13-18]. In our previous work [5], we demonstrated the possibility of implementation of the OTSs localized at the edges of a one-dimensional (1D) PC bounded by metal-dielectric isotropic nanocomposite media on its one of both sides. The nanocomposite consists of spherical or spheroidal metal nanoparticles dispersed in a transparent matrix and is characterized by the effective permittivity $\varepsilon(\omega)$; the optical characteristics of the initial materials does not exhibit resonance features. The position of a frequency range where the nanocomposite behaves like a metal, i.e., $\operatorname{Re}(\varepsilon(\omega))<0$, depends on permittivities of the initial materials and nanoparticle concentration and shape, which opens new possibilities for controlling the optical properties of the OTSs by varying the nanocomposite parameters.

The aim of this study was to investigate the spectral properties of the OTSs localized at the edges of a 1D photonic crystal bounded by a nanocomposite consisting of spherical nanoparticles with a dielectric core and a metal shell, which are dispersed in a transparent matrix, on one or both sides. The spectral properties of the PC are studied using the transfer matrix method.

\section{Description of the model}

The investigated PC structure is a layered medium bounded by a nanocomposite layer on its one or both sides (figure 1). The nanocomposite layer with the thickness $\mathrm{W}_{\mathrm{d}}=150 \mathrm{~nm}$ consists of shelled spherical nanoparticles uniformly distributed in a dielectric matrix made of transparent optical glass with the permittivity $\varepsilon_{\mathrm{m}}=2.56$. The alternating layers that form a PC unit cell are zirconium dioxide $\mathrm{ZrO}_{2}$ with the permittivity $\varepsilon_{\mathrm{a}}=$ 4.16 and silicon dioxide $\mathrm{SiO}_{2}$ with the permittivity $\varepsilon_{\mathrm{b}}=2.10$; the respective layer thicknesses are $\mathrm{W}_{\mathrm{a}}=50 \mathrm{~nm}$ and $\mathrm{W}_{\mathrm{b}}=74 \mathrm{~nm}$. The investigated PC structure is placed in a medium (air) with a permittivity of unity and consists of $\mathrm{N}=16$ or 17 layers for one and two nanocomposite layers bounding the PC, respectively.

The effective permittivity of the nanocomposite is determined from the Maxwell-Garnett formula [18] widely used in studying composite media. Generalization of this formula to nanocomposites with dispersed shelled spherical particles yields the effective permittivity [19]

$$
\varepsilon=\varepsilon_{\mathrm{m}}\left(1+\frac{3 \mathrm{f} \alpha}{1-\mathrm{f \alpha} \alpha}\right)
$$

where $\mathrm{f}$ is the filling factor, i.e., the fraction of nanoparticles in the matrix and $\alpha$ is the parameter proportional to polarizability of the layered nanoparticle. The Maxwell-Garnett model for the effective medium suggests the quasi-static consideration of an electrodynamically isotropic nanocomposite layer with the size of nanoparticles and distance between them much smaller than the wavelength of light in the effective medium. 
For a spherical particle with core permittivity $\varepsilon_{\mathrm{c}}$ and shell permittivity $\varepsilon_{\mathrm{s}}$, which is placed in a medium with permittivity $\varepsilon_{\mathrm{m}}$, parameter $\alpha$ is determined as [20]

$$
\alpha=\frac{\left(\varepsilon_{\mathrm{s}}-\varepsilon_{\mathrm{m}}\right)\left(\varepsilon_{\mathrm{c}}+2 \varepsilon_{\mathrm{s}}\right)+\beta\left(\varepsilon_{\mathrm{m}}+2 \varepsilon_{\mathrm{s}}\right)\left(\varepsilon_{\mathrm{c}}-\varepsilon_{\mathrm{s}}\right)}{\left(\varepsilon_{\mathrm{s}}+2 \varepsilon_{\mathrm{m}}\right)\left(\varepsilon_{\mathrm{c}}+2 \varepsilon_{\mathrm{s}}\right)+2 \beta\left(\varepsilon_{\mathrm{s}}-\varepsilon_{\mathrm{m}}\right)\left(\varepsilon_{\mathrm{c}}-\varepsilon_{\mathrm{s}}\right)},
$$

where $\beta$ is the ratio between the particle core volume and the total particle volume. In the investigated structure, nanoparticles consist of the dielectric core with the permittivity $\varepsilon_{\mathrm{c}}=3$ and the silver shell with permittivity $\varepsilon_{\mathrm{s}}$ determined from the Drude-Sommerfeld formula

$$
\varepsilon_{\mathrm{S}}(\omega)=\varepsilon_{0}-\frac{\omega_{\mathrm{p}}^{2}}{\omega(\omega+\mathrm{i} \gamma)},
$$

where $\varepsilon_{0}$ is the constant that makes allowance for the contributions of interband transitions of bound electrons; $\omega_{\mathrm{p}}$ is the plasma frequency; $\gamma$ is the damping coefficient, i.e., the quantity reciprocal to the electron relaxation time; and $\omega$ is the frequency of incident light. For silver, we have $\varepsilon_{0}=5, \hbar \omega_{\mathrm{p}}=9 \mathrm{eV}$, and $\hbar \gamma=0.02 \mathrm{eV}$. At present, there exists the nanoparticle fabrication technology, which allows varying the core and shell sizes in wide ranges $[21,22]$.

Figure 2 shows the dependence of the effective nanocomposite permittivity on the wavelength of incident light for the chosen parameters of the nanocomposite. One can see two resonance permittivity portions associated with the plasmon resonance of nanoparticles. Surface plasmons arise at the metal shell/core and metal shell/matrix interfaces. As the core permittivity is increased, the short-wavelength resonance permittivity portion sharply grows, whereas the long-wavelength portion slightly decreases. The opposite situation is observed with an increase in the matrix permittivity. In addition, the both resonances shift toward longer wavelengths. As the shell becomes thinner, the coupling between plasmons localized at the shell boundaries becomes stronger and the short-wavelength resonance permittivity portion shifts toward shorter wavelengths, while the long-wavelength portion shifts toward longer wavelengths.

The transmission, reflection, and absorption spectra of the layered structure were calculated using the transfer matrix method [23] for the plane light wave normally incident onto the structure.

\section{Results and discussion}

Figure 3 shows transmission, reflection, and absorption spectra for the PC consisting of 15 layers, which is bounded by the nanocomposite layer on its one side without nanoparticles and with a nanoparticle fraction of 0.3 in the nanocomposite. It can be seen that the spectra qualitatively change upon embedding of nanoparticles. At a wavelength of about $348 \mathrm{~nm}$, the stopband forms (figure 3a), which is associated with absorption of light at the resonance wavelength (figure 3c) corresponding to the short-wavelength resonance portion of the nanocomposite permittivity (figure 2). The initial band gap in the transmission spectrum almost doubles due to the high reflection of light (figure 3b) at these wavelengths, which is related to the large negative value of the real part of the nanocomposite permittivity (figure 2). In addition, near the short-wavelength boundary of the band gap, the transmission peak arises, which is related to the occurrence of the OTS. The light field in the Tamm plasmon polariton is localized in the region comparable with the wavelength. The local field intensity distribution at a wavelength of $391 \mathrm{~nm}$ corresponding to the OTS is shown in figure $3 \mathrm{~d}$. The field is localized at the $\mathrm{PC} /$ nanocomposite interface, since at this wavelength the real part of the nanocomposite permittivity is 
negative and the nanocomposite behaves like a metal (figure 2); therefore, the field penetrates into it only to the skin layer depth. Deeper in the PC, the intensity envelope exponentially decreases due to the Bragg diffraction, since this wavelength lies within the PC band gap.

As the nanocomposite film thickness is increased, the transmission and local field intensity corresponding to the OTS decrease. In this case, the reflection of light enhances and the absorption weakens. The transmission peak shifts toward longer wavelengths. At a film thickness of $200 \mathrm{~nm}$, the transmission at an OTS wavelength of $391.3 \mathrm{~nm}$ decreases by $15 \%$, the absorption decreases by $13 \%$, the reflection increases by $28 \%$, and the local intensity drops by $30 \%$.

However, an increase in the filling factor at the film thickness $\mathrm{W}_{\mathrm{d}}=150 \mathrm{~nm}$ leads to the slight transmission growth and a decrease in the local field intensity at the OTS wavelength. Then, the reflection of light increases and the absorption decreases. This is due to the fact that with increasing filling factor the imaginary part of the nanocomposite permittivity at the OTS wavelength decreases. The transmission peak shifts toward shorter wavelengths. At a filling factor of 0.4, the transmission at an OTS wavelength of $385.6 \mathrm{~nm}$ increases by $3 \%$, the absorption decreases by $20 \%$, the reflection increases by $17 \%$, and the local intensity decreases by $17 \%$.

As the ratio between the core and shell radii grows at the invariable initial parameters, the transmission and local field intensity at the OTS wavelength decrease. In this case, the reflection weakens, while the absorption grows. The transmission peak shifts toward longer wavelengths. With an increase in the ratio between the core and shell radii to 0.4 , the transmission at an OTS wavelength of $398 \mathrm{~nm}$ decreases by $12 \%$, the absorption increases by $8 \%$, the reflection increases by $4 \%$, and the local intensity decreases by $11 \%$.

Figure 4 shows transmission, reflection, and absorption spectra of the PC consisting of 15 layers and bounded on its one or both sides by the nanocomposite layer with a filling factor of 0.3 . When the second nanocomposite layer is introduced, the peak in the PC band gap in the transmission spectrum splits by $6.3 \mathrm{~nm}$. This splitting of the resonance Tamm wavelength is caused by elimination of degeneracy due to the interference of the optical Tamm modes localized at the interfaces. The stopband related to the absorption in the shortwavelength spectral range remains almost invariable.

Figure 5 shows the electric field strength distribution over the structure at the wavelengths corresponding to the split peaks. It can be seen that the coupling of the optical Tamm plasmons localized at the $\mathrm{PC} /$ nanocomposite interfaces results in the formation of symmetric and antisymmetric waveguide modes. As in study [24], for the PC bounded by silver films we obtained the field distribution similar to the field distribution in the symmetric and antisymmetric modes in figure 5.

As the nanocomposite film thickness is increased to $200 \mathrm{~nm}$, the transmission and local field intensity at the OTS wavelengths decrease. The absorption slightly grows at the wavelength of the short-wavelength bound plasmon polariton and slightly decreases at the wavelength of the long-wavelength one. The reflection of light significantly increases for the both bound plasmon polaritons. The position of the OTS peaks and the distance between them remain almost invariable.

As the filling factor increases to 0.4 , the transmission weakens and the local field intensity for the OTS peaks increases. In this case, the absorption slightly increases for the short-wavelength bound plasmon polariton 
and slightly decreases for the long-wavelength one. The reflection of light significantly grows for the both bound plasmon polaritons. The peaks corresponding to the OTSs shift toward shorter wavelengths and the distance between them increases to $8.6 \mathrm{~nm}$.

With an increase in the ratio between the core and shell radii to 0.35 , the transmission and local field intensity for the peaks corresponding to the OTSs decrease. In this case, the absorption slightly decreases for the short-wavelength bound plasmon polariton and slightly increases for the long-wavelength one. The reflection of light decreases for the both bound plasmon polaritons. The peaks corresponding to the OTSs shift toward longer wavelengths and the distance between them decreases to $5 \mathrm{~nm}$.

Varying the parameters of the structure, one can obtain coincidence of the two regions with the negative real part of the nanocomposite permittivity with the two corresponding band gaps in the seed transmission spectrum of the PC ( $\mathrm{f}=0$ ) (figure 6a). In this case, after embedding of nanoparticles in the nanocomposite, the OTS peak arises at the boundary of the both band gaps (figure 6b). The local field intensity distribution at wavelengths of 319.2 and $858.8 \mathrm{~nm}$ corresponding to the OTSs is presented in figure 7 . It can be seen that the field is localized at the PC/nanocomposite interface for the both peaks.

\section{Conclusions}

The spectral properties of a 1D PC bounded by the nanocomposite layer on its one or both sides were investigated. The nanocomposite consists of spherical nanoparticles with a dielectric core and a silver shell, which are dispersed in transparent optical glass.

It was demonstrated that the transmission spectrum of the investigated structure can simultaneously contain the stopband related to absorption at the resonance frequency of the nanocomposite permittivity and the transmission peak in the PC band gap corresponding to the OTS caused by the negative real part of the nanocomposite permittivity.

It was observed that for the PC bounded by the nanocomposite layers on its both sides, the peak corresponding to the OTS splits at the formation of bound Tamm plasmon polaritons, whereas the stopband remains almost invariable.

It was established that the characteristics of the Tamm states localized at the edge of a PC significantly depend on the concentration of nanoparticles in the nanocomposite film, film thickness, and ratio between the particle core volume and the total particle volume.

It was shown that at certain parameters of the structure, two Tamm states can manifest themselves in two band gaps of the PC. This can occur when the regions of negative real part of the nanocomposite permittivity coincide with the two band gaps in the PC seed transmission spectrum.

\section{Acknowledgments}

This study was supported by the Ministry of Education and Science of the Russian Federation, state order no. 3.1276.2014/K for the Siberian Federal University in 2015, the Russian Foundation for Basic Research, project no. 14-02-31248, and the joint research project of the Siberian Branch of the Russian Academy of Sciences and the Ministry of Science and Technology of the Republic of China. 


\section{References}

1. Vinogradov A P, Dorofeenko A V, Merzlikin A M and Lisyansky A A 2010 Surface states in photonic crystals Phys. Usp. 53 243-56

2. Kavokin A, Shelykh I and Malpuech G 2005 Optical Tamm states for the fabrication of polariton lasers Appl. Phys. Lett. 87261105

3. Kavokin A V, Shelykh I A and Malpuech G 2005 Lossless interface modes at the boundary between two periodic dielectric structures Phys. Rev. B 72233102

4. Kaliteevski M, Iorsh I, Brand S, Abram R A, Chamberlain J M, Kavokin A V and Shelykh I A 2007

Tamm plasmon-polaritons: Possible electromagnetic states at the interface of a metal and a dielectric Bragg mirror Phys. Rev. B 76165415

5. Vetrov S Ya, Bikbaev R G and Timofeev I V 2013 Optical Tamm States at the Interface between a Photonic Crystal and a Nanocomposite with Resonance Dispersion JETP 117 988-98

6. Sasin M E, Seisyan R P, Kalitteevski M A, Brand S, Abram R A, Chamberlain J M, Egorov A Yu, Vasil'ev A P, Mikhrin V S and Kavokin A V 2008 Tamm plasmon polaritons: Slow and spatially compact light Appl. Phys. Lett. 92, 251112

7. Goto T, Dorofeenko A V, Merzlikin A M, Baryshev A V, Vinogradov A P, Inoue M, Lisyansky A A and Granovsky A B 2008 Optical Tamm States in One-Dimensional Magnetophotonic Structures Phys. Rev. Lett. 101113902

8. Zhang W L and Yu S F 2010 Bistable switching using an optical Tamm cavity with a Kerr medium Opt. Commun. $2832622-6$

9. Zhou H, Yang G, Wang K, Long H and Lu P 2010 Multiple optical Tamm states at a metal-dielectric mirror interface Opt. Lett. 35 4112-4

10. Vinogradov A P, Dorofeenko A V, Erokhin S G, Inoue M, Lisyansky A A, Merzlikin A M and Granovsky A B 2006 Surface state peculiarities in one-dimensional photonic crystal interfaces Phys. Rev. B 74 045128

11. Zhang X-L, Song J-F, Li X-B, Feng J and Sun H-B 2012 Optical Tamm states enhanced broad-band absorption of organic solar cells Appl. Phys. Lett.101 243901

12. Gong Y, Liu X, Lu H, Wang L and Wang G 2011 Perfect absorber supported by optical Tamm states in plasmonic waveguide Opt. Exp. 19 18393-8

13. Tikhodeev S G and Gippius N A 2009 The scattering matrix and optical properties of metamaterials Phys. Usp. 179 1027-30

14. D'yachenko P N and Miklyaev Yu V 2007 Dimensional photonic crystals based on nanocomposite: metal nanoparticles - dielectric Computer Optics 31 31-4

15. Vetrov S Ya, Avdeeva A Yu and Timofeev I V 2011 Spectral properties of a one-dimensional photonic crystal with a resonant defect nanocomposite layer JETP 113 755-61

16. Vetrov S Ya, Pankin P S and Timofeev I V 2014 Peculiarities of spectral properties of a onedimensional photonic crystal with an anisotropic defect layer of the nanocomposite with resonant dispersion Quantum Electron $\mathbf{4 4} 881-4$ 
17. Moiseev S G, Ostatochnikov V A and Sementsov D I 2012 Defect mode suppression in a photonic crystal structure with a resonance nanocomposite layer Quantum Electronics $\mathbf{4 2}$ 557-60

18. Maxwell Garnett J C 1904 Colours in metal glasses and in metallic films Phil. Trans. R. Soc. Lond. $203385-420$

19. Sihvola A 2008 Electromagnetic Mixing Formulas and Applications (London: Institution of Engineering and Technology)

20. Klimov V 2014 Nanoplasmonics (Boca Raton: CRC Press)

21. Oldenburg S J, Averitt R D, Westcott S L and Halas N J 1998 Nanoengineering of optical resonances Chem. Phys. Lett. 288 243-7

22. Wong H, Wu Y, Lassiter B, Nehl C L, Hafner J H, Nordlander P and Halas N J 2006 Symmetry breaking in individual plasmonic nanoparticles PNAS 103 10856-60

23. Yeh P 1979 Electromagnetic propagation in birefringent layered media J. Opt. Soc. Amer. 69 742-56

24. Iorsh I, Panicheva P V, Slovinskii I A and Kaliteevskii M A 2012 Coupled Tamm plasmons Technical Physics Letters 38 351-3 


\section{Figure captions}

Figure 1. Schematic of a 1D PC bounded by a nanocomposite layer.

Figure 2. Real $\varepsilon^{\prime}$ (thin curve) and imaginary $\varepsilon^{\prime \prime}$ (bold curve) portions of the nanocomposite permittivity vs wavelength of incident light. Insert: enlarged framed portion. The parameters are $f=0.3, \beta=(0.3)^{3}, \varepsilon_{\mathrm{m}}=2.56$, and $\varepsilon_{\mathrm{c}}=3$.

Figure 3. (a) Transmittance, (b) reflectance, and (c) absorbance of the PC bounded by the nanocomposite layer vs wavelength of the incident light at the filling factors $f=0$ (thin curve) and $f=0.3$ (bold curve). (d) Spatial distribution of the local field intensity at a wavelength of $391 \mathrm{~nm}(\mathrm{f}=0.3)$ (bold curve) and refractive indices of the layers (thin curve).

Figure 4. (a) Transmittance, (b) reflectance, and (c) absorbance of the PC bounded on one (thin curve) and both sides (bold line) by the nanocomposite layer vs wavelength of the incident light at $\mathrm{W}_{\mathrm{d}}=150 \mathrm{~nm}$ and $\mathrm{f}=0.3$.

Figure 5. Spatial distribution of the electric field strength and refractive indices of the PC layers for (a) the short-wavelength $(387.9 \mathrm{~nm})$ and (b) long-wavelength $(394.2 \mathrm{~nm})$ plasmon polariton modes of the structure at $\mathrm{W}_{\mathrm{d}}=150 \mathrm{~nm}$ and $\mathrm{f}=0.3$.

Figure 6. Transmittance of the PC bounded by the nanocomposite layer vs wavelength of the incident light. The bold line in figure $6 \mathrm{a}$ corresponds to the normalized real part of the nanocomposite permittivity. The parameters are $\mathrm{W}_{\mathrm{d}}=340.5 \mathrm{~nm}, \mathrm{~W}_{\mathrm{a}}=113.5 \mathrm{~nm}, \mathrm{~W}_{\mathrm{b}}=168 \mathrm{~nm}, \beta=(0.82)^{3}, \varepsilon_{\mathrm{m}}=4, \varepsilon_{\mathrm{c}}=10, \mathrm{f}=0$ (thin curve), and $\mathrm{f}=0.4$ (bold curve).

Figure 7. Spatial distribution of the local field intensity for the peaks corresponding to the OTSs in figure $6 \mathrm{~b}$ (bold curve) and refractive indices of the layers (thin curve). The wavelengths are (a) 319.2 and (b) $858.8 \mathrm{~nm}$. 
Figures

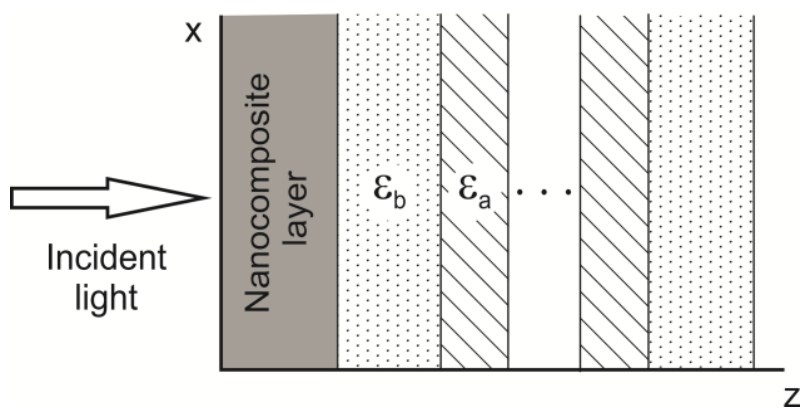

Figure 1. Schematic of a 1D PC bounded by a nanocomposite layer.

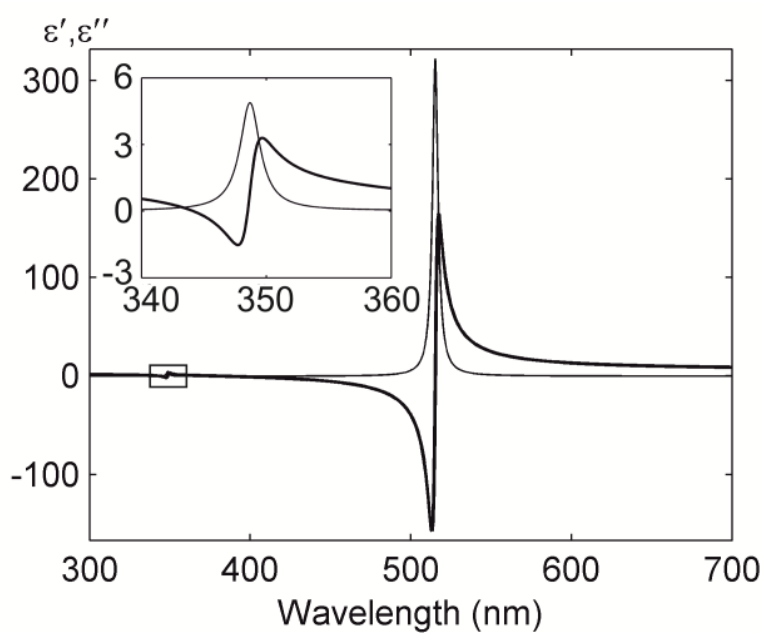

Figure 2. Real $\varepsilon^{\prime}$ (thin curve) and imaginary $\varepsilon^{\prime \prime}$ (bold curve) portions of the nanocomposite permittivity vs wavelength of incident light. Insert: enlarged framed portion. The parameters are $f=0.3, \beta=(0.3)^{3}, \varepsilon_{\mathrm{m}}=2.56$, and $\varepsilon_{\mathrm{c}}=3$. 

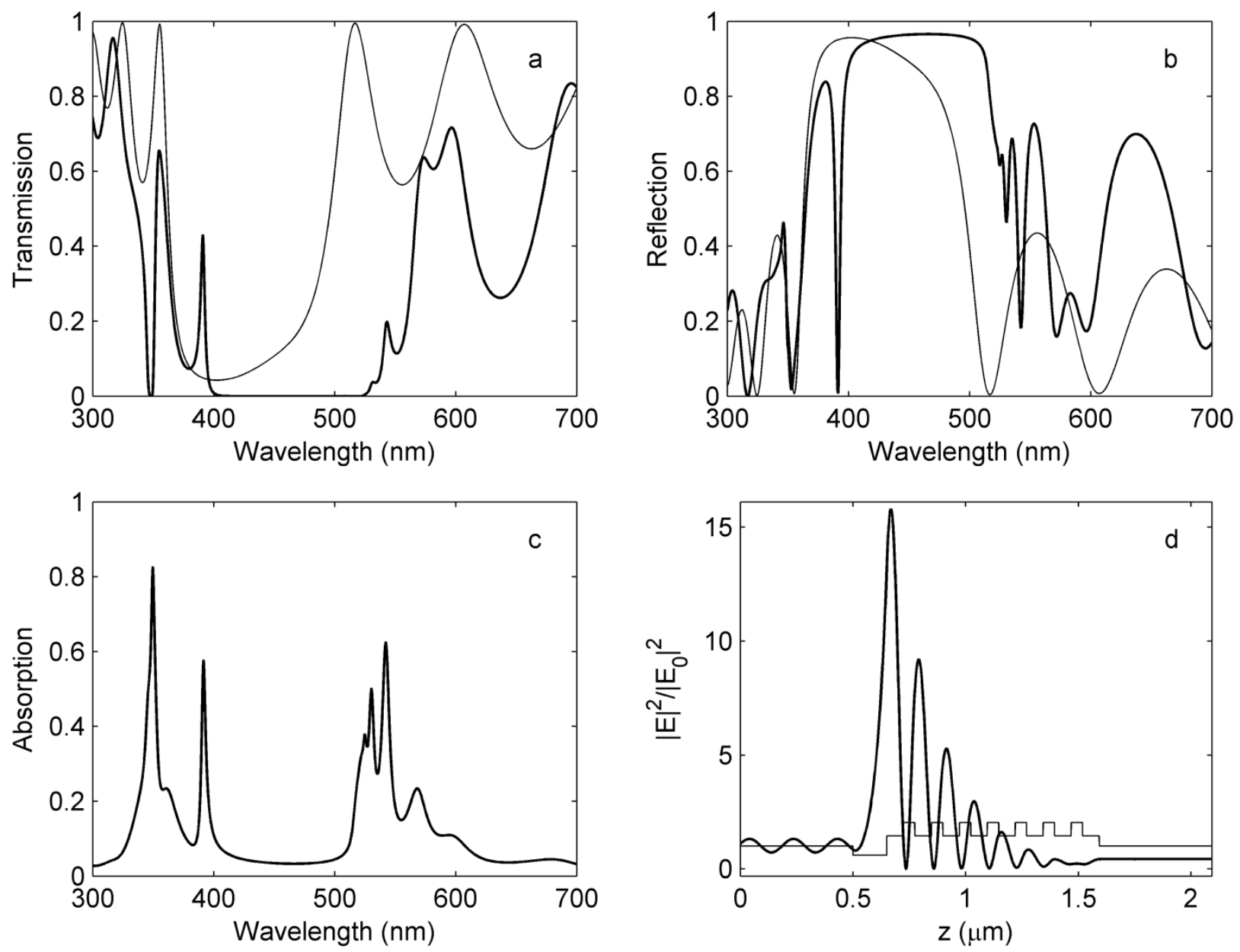

Figure 3. (a) Transmittance, (b) reflectance, and (c) absorbance of the PC bounded by the nanocomposite layer vs wavelength of the incident light at the filling factors $f=0$ (thin curve) and $f=0.3$ (bold curve). (d) Spatial distribution of the local field intensity at a wavelength of $391 \mathrm{~nm}(\mathrm{f}=0.3)$ (bold curve) and refractive indices of the layers (thin curve). 

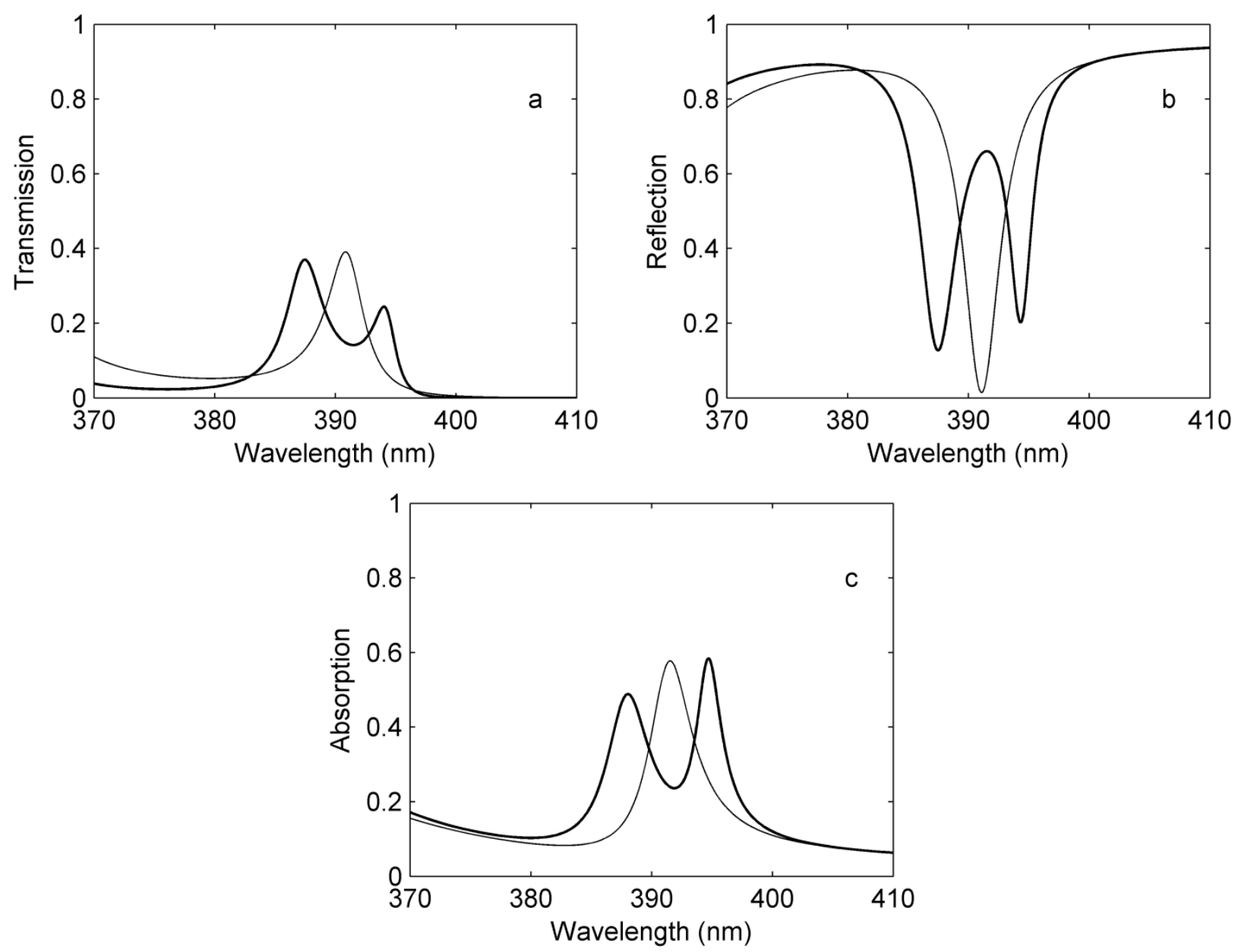

Figure 4. (a) Transmittance, (b) reflectance, and (c) absorbance of the PC bounded on one (thin curve) and both sides (bold line) by the nanocomposite layer vs wavelength of the incident light at $\mathrm{W}_{\mathrm{d}}=150 \mathrm{~nm}$ and $\mathrm{f}=0.3$.
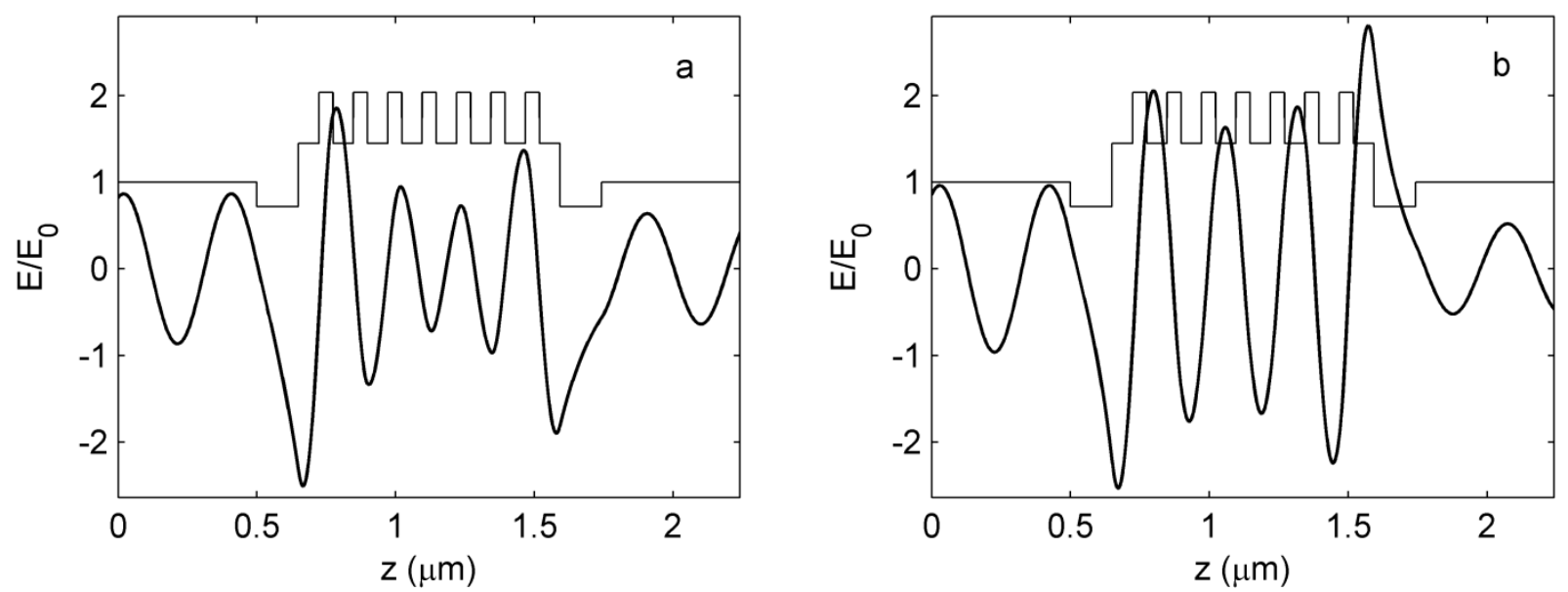

Figure 5. Spatial distribution of the electric field strength and refractive indices of the PC layers for (a) the short-wavelength $(387.9 \mathrm{~nm})$ and $(\mathrm{b})$ long-wavelength $(394.2 \mathrm{~nm})$ plasmon polariton modes of the structure at $\mathrm{W}_{\mathrm{d}}=150 \mathrm{~nm}$ and $\mathrm{f}=0.3$. 

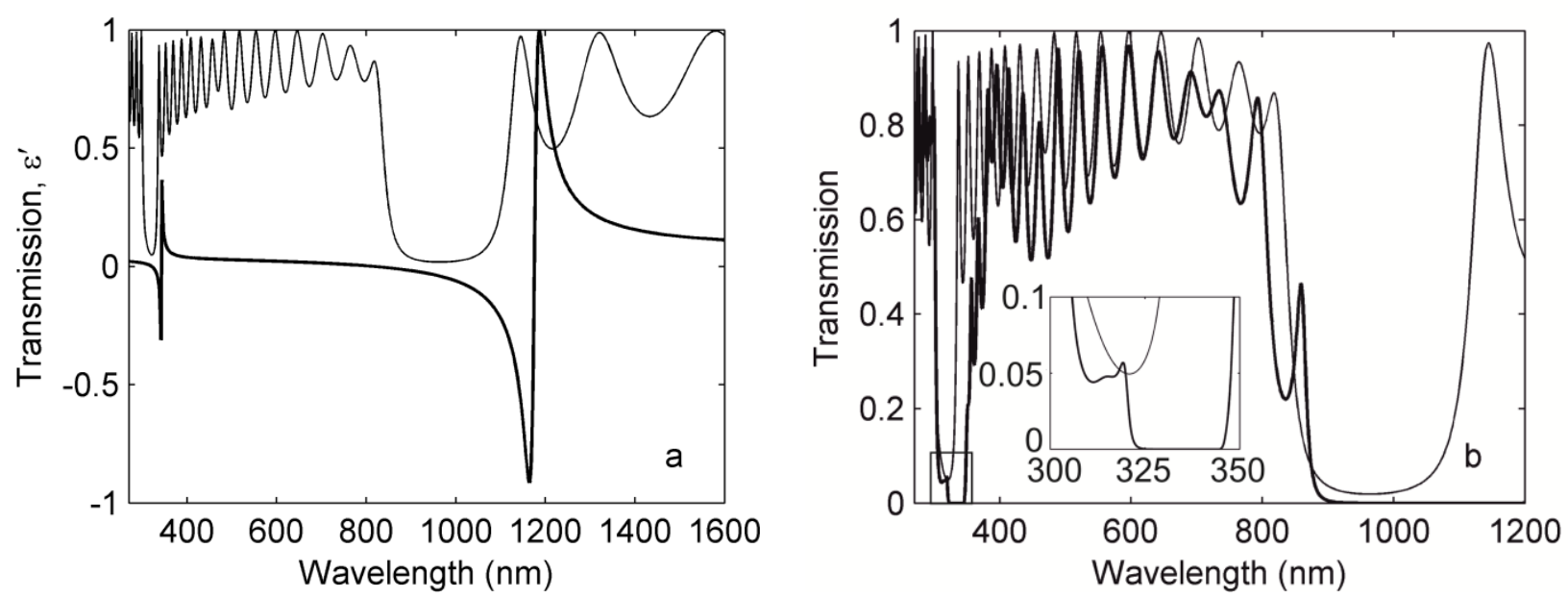

Figure 6. Transmittance of the PC bounded by the nanocomposite layer vs wavelength of the incident light. The bold line in figure 6a corresponds to the normalized real part of the nanocomposite permittivity. The parameters are $\mathrm{W}_{\mathrm{d}}=340.5 \mathrm{~nm}, \mathrm{~W}_{\mathrm{a}}=113.5 \mathrm{~nm}, \mathrm{~W}_{\mathrm{b}}=168 \mathrm{~nm}, \beta=(0.82)^{3}, \varepsilon_{\mathrm{m}}=4, \varepsilon_{\mathrm{c}}=10, \mathrm{f}=0$ (thin curve), and $\mathrm{f}=0.4$ (bold curve).
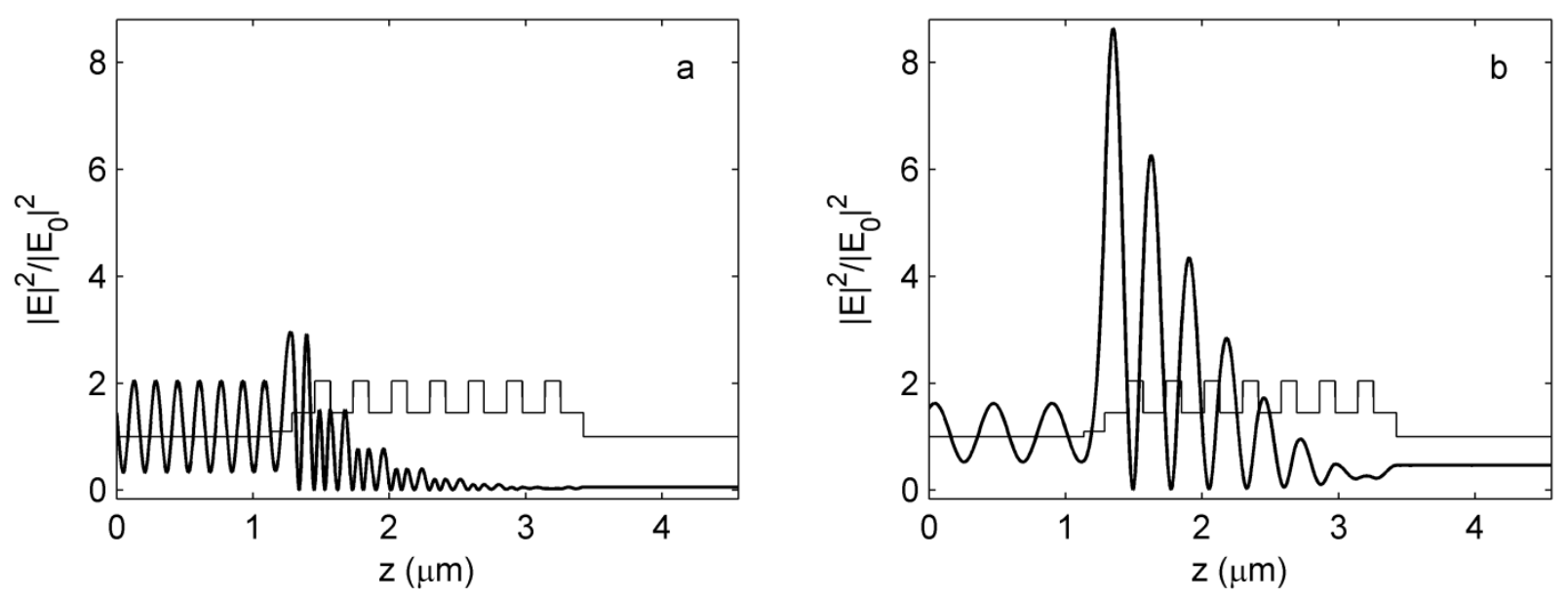

Figure 7. Spatial distribution of the local field intensity for the peaks corresponding to the OTSs in figure $6 \mathrm{~b}$ (bold curve) and refractive indices of the layers (thin curve). The wavelengths are (a) 319.2 and (b) $858.8 \mathrm{~nm}$. 